\title{
TAGUNG
}

\section{Das Autonomiestreben nationaler Exekutiven in der europäischen Justiz- und Innenpolitik}

\author{
Marianne Haase*
}

Die Vergemeinschaftung der Politik der „Freiheit, der Sicherheit und des Rechts“ hat bedeutend zugenommen, obwohl insbesondere mit sicherheitspolitischen Themen Kernkompetenzen nationalstaatlicher Souveränität berührt werden. Inhaltlich ist ein tendenziell restriktiver Ansatz innerhalb der europäischen Justiz- und Innenpolitik zu verzeichnen.

Der Intergouvernementalismus bedient sich eines rationalistischen Analyserahmens, um solche Integrationsprozesse zu erklären. Demnach sind es Akteure in Form von Staaten, die ihre jeweilige Verhandlungsmacht nutzen, um politische Ziele zu verfolgen. Die ,venue-shopping'-These, welche von Virginie Guiraudon $^{1}$ auf das Politikfeld der europäischen Justiz- und Innenpolitik angewandt wurde, erklärt die Integration und gleichzeitige Dominanz des restriktiven Ansatzes durch die Annahme, dass nationale Akteure versuchen, Handlungsautonomie zu erlangen, welche ihnen auf nationalstaatlicher Ebene nicht gegeben wäre. Neben der Supranationalisierung treten zudem vermehrt exklusive und exekutivdominierte Kooperationen auf, die es zu erklären gilt.

Ziel der Tagung war es, die Entwicklung und den Charakter der europäischen Justiz- und Innenpolitik anhand folgender Fragen zu erfassen: Trifft die ,venue-shopping'-These (immer noch) für die europäische Justiz- und Innenpolitik $\mathrm{zu}$ oder sind es andere theoretische Ansätze wie das Multilevel-Game nach

Die Europäisierung der Kontrolle:
Deutschland und die Schaffung eines
Raums der Freiheit, der Sicherheit und
$\quad$ des Rechts
Workshop der Stiftung Wissenschaft und Politik
(SWP), des Zentralinstituts für Regionen-
forschung der Friedrich-Alexander-Universität
Erlangen-Nürnberg, der Universität Bielefeld,
der Friedrich-Ebert-Stiftung, des Arbeitskreises
Europäische Integration e.V. mit Unterstützung
$\quad$ der Europäischen Kommission
$\quad$ Berlin, 23. Mai 2008
Begrüßung
PD Dr. Petra BENDEL, Zentralinstitut für Regi-
onenforschung, Friedrich-Alexander-Universität
Erlangen-Nürnberg
Günther SCHULTZE, Friedrich-Ebert-Stiftung,
Bonn
Die ,venue-shopping“-These: Ein Erklärungs-
ansatz für die europäische Zusammenarbeit?
Vorsitz
PD Dr. Petra BENDEL, Zentralinstitut für Regi-
onenforschung, Friedrich-Alexander-Universität
Erlangen-Nürnberg
Venue-shopping and JHA: still relevant?
Prof. Dr. Virginie GUIRAUDON, Centre d'Étu-
des et de Recherches Administratives, Politiques
et Sociales (CERAPS), Université de Lille 2
Zwei-Ebenen-Spiele in der EU Zusammenarbeit?
Das Beispiel der Asylpolitik
Dorothee LAUTER, Freie Universität Berlin
Dr. Arne NIEMANN, Universiteit van Amsterdam
Die Prümer Methode: Schengen II oder Zwei-
Ebenen-Spiele?
Daniela KIETZ, Stiftung Wissenschaft und Poli-
tik, Berlin

Die Europäisierung der Kontrolle: Deutschland und die Schaffung eines des Rechts

orkshop der Stiftung Wissenschaft und Politik forschung der Friedrich-Alexander-Universität Erlangen-Nürnberg, der Universität Bielefeld, der Friedrich-Ebert-Stiftung, des Arbeitskreises der Europäischen Kommission

$$
\text { Berlin, 23. Mai } 2008
$$

\section{Begrüßung}

PD Dr. Petra BENDEL, Zentralinstitut für Regionenforschung, Friedrich-Alexander-Universität Erlangen-Nürnberg

nther SCHULTZE, Friedrich-Ebert-Stiftung,

Die ,venue-shopping“"These: Ein Erklärungsansatz für die europäische Zusammenarbeit? Vorsitz onenforschung, Friedrich-Alexander-Universität Prof. Dr. Virginie GUIRAUDON, Centre d'Études et de Recherches Administratives, Politiques

Das Beispiel der Asylpolitik

Dorothee LAUTER, Freie Universität Berlin

Die Prümer Methode: Schengen II oder ZweiDaniela KIETZ, Stiftung Wissenschaft und PoliBerlin

* Marianne Haase, Zentralinstitut für Regionenforschung, Friedrich-Alexander-Universität Erlangen-Nürnberg.

1 Virginie Guiraudon: European Integration and Migration Policy: Vertical Policy-making as Venue shopping, in: Journal of Common Market Studies 2/2000, S. 251-271. 
Putnam, ${ }^{2}$ welche für die Analyse herangezogen werden müssten? Wie interagieren die europäische und die nationalstaatliche Ebene, welche Transferprozesse lassen sich beobachten? Welche Integrationsarten herrschen vor und wie lassen sich Formen der differenzierten Integration bei gleichzeitiger Supranationalisierung erklären? Wie können die europäische Migrations- und Asylpolitik, der europäische Datenschutz im Bereich Justiz und Inneres und die europäische Politik der inneren Sicherheit charakterisiert werden? Welche Strategie verfolgt Deutschland? Erweisen sich die theoretischen Analyseinstrumente als angemessen oder ergänzungsbedürftig?

Enge nationale Handlungsspielräume als Erklärungsansatz für die Integration der Justizund Innenpolitik

Im ersten Panel ging Virginie Guiraudon der Frage nach, ob die von ihr vertretene, venueshopping '-These für den Bereich der Justizund Innenpolitik immer noch relevant sei. Kernstück dieser These ist die Annahme, dass die Innen- und Justizminister der Mitgliedstaaten in ihrer Eigenschaft als Rationalisten, die für sie jeweils günstigste Handlungsebene (,venue') suchen, um ihr politisches Ziel zu erreichen. Im Bereich der europäischen Justiz- und Innenpolitik stellen auf nationalstaatlicher Ebene insbesondere nationale Gerichte, konkurrierende Ministerien, aber auch Nichtregierungsorganisationen Einschränkungen für eine eher restriktiv ausgelegte Justiz- und Innenpolitik dar. Minister sind bestrebt, diese Einschränkungen $\mathrm{zu}$ umgehen und weichen aus diesem Grund auf die europäische Handlungsebene aus. Nach Guiraudon zeigt sich dieses Handlungsmuster in der Vergemeinschaftung der Justiz- und Innenpolitik. Als konkretes Beispiel hierfür führte sie den Aufbau des Schengener Raumes an. Doch sei, so konstatierte Guiraudon, dieses Vorgehen auch auf europäischer Ebene aufgrund der Instituti-
Die Orientierung der EU-Politik: Triumphiert tatsächlich der kontrollorientierte Ansatz?

Vorsitz

PD Dr. Sabine RIEDEL, Stiftung Wissenschaft und Politik, Berlin

Die EU und die temporäre Arbeitsmigration: Mittel der Migrationskontrolle oder der Entwicklungspolitik?

Dr. Hendrik CREMER, Deutsches Institut für Menschenrechte, Berlin

EU-Datenschutzstandards: Liberalismus vs. Kontrolle?

Marie McGINLEY, Europäische Investitionsbank, Luxemburg

Menschenrechtsstandards an und jenseits der gemeinsamen EU-Außengrenze

Dr. Ruth WEINZIERL, Deutsches Institut für Menschenrechte, Berlin

Die EU-Zusammenarbeit und die Ausdehnung der Macht der Exekutive: Der Fall Deutschland

Vorsitz

Andreas ETTE, Bundesinstitut für Bevöl-

kerungsforschung, Wiesbaden

Deutschland, Frontex und die Versetzung der deutschen Grenzen

Dr. Mechthild BAUMANN, Institut für Migrations- und Sicherheitsstudien, Berlin

The German executive as venue-shopper in Justice and Home Affairs?

Prof. Dr. Simon BULMER, University of Sheffield

German anti-terror policy: human rights protection in the European context

Dr. Wilhelm KNELANGEN, Christian-AlbrechtsUniversität zu Kiel

Zur Ausdehnung des wissenschaftlichen Werkzeugs: Wie weit reichen gängige Erklärungsansätze?

Vorsitz

PD Dr. Petra BENDEL, Zentralinstitut für Regionenforschung, Friedrich-Alexander-Universität Erlangen-Nürnberg

Jenseits von „uploads“ und Anpassungen - die Hartnäckigkeit der nationalen Regierungen in JI konzeptualisieren

Prof. Dr. Timm BEICHELT, Europa-Universität Viadrina, Frankfurt (Oder)

2 Robert Putnam: Diplomacy and Domestic Politics: The Logic of Two-level Games, in: International Organization 3/1988, S. 427-460. 
onalisierung und Politisierung des Politikfeldes zunehmend durch Handlungsbeschränkungen gekennzeichnet. So hätten insbesondere nach dem Inkrafttreten des Amsterdamer Vertrags (1999) - und erneut bei etwaigem Inkrafttreten des Vertrags von Lissabon - das Europäische Parlament, die nationalen Parlamente sowie der Europäische Gerichtshof Kompetenzen hinzugewonnen. So erscheine es fraglich, ob sich die europäische Ebene auch weiterhin als günstig für die Durchsetzung einer eher restriktiven Justizund Innenpolitik erweisen könne. Zudem würden vermehrt qualifizierte Mehrheitsentscheidungen getroffen, die die Verhandlungsmacht von nationalstaatlichen Akteuren einschränkten. Die momentane Entwicklung tendiere vor diesem Hintergrund zu intensiven transgouvernementalen Handlungsstrategien. Diese hielten weiterhin ,Exit-Strategien bereit. Flexible Integrationsmechanismen, die nicht die gesamte EU-27 einbeziehen, seien als Fortsetzung der ,Schengener Club-Mentalität‘ zu werten. Als Beispiel nannte Guiraudon den Fall des Prümer Vertrags, der erst expost in den EU-Rahmen eingefügt wurde. Besonders häufig seien zudem auch operationelle Kooperationen, wie sie zum Beispiel durch FRONTEX praktiziert würden. Ebenso sei zu beobachten, dass migrationsrelevante Aspekte in die externen Beziehungen der Europäischen Union zu Drittstaaten Eingang gefunden hätten, was ebenso ,venue-shopping ‘ darstelle. Guiraudon wies jedoch auch daraufhin, dass die Kosten für die Akteure des ,venue-shopping' nicht zu unterschätzen seien und führte dabei insbesondere finanzielle $\mathrm{Be}$ lastungen und schlechte Effizienz an. Darüber hinaus drohe die Glaubwürdigkeit der Europäischen Union hinsichtlich der Wahrung menschenrechtlicher Ansprüche zu leiden.

Am Beispiel der europäischen und deutschen Asylpolitik analysierten Dorothee Lauter und Arne Niemann sowohl ,top-down'- als auch ,bottom-up'-Prozesse der Europäisierung und
Ungleiche Europäisierung: Negative und positive Integration im europäischen Regieren des Politikfeldes innere Sicherheit

Dr. Wolfgang WAGNER, Vrije Universiteit Amsterdam

\section{Schlussworte}

PD Dr. Petra BENDEL, Zentralinstitut für Regionenforschung, Friedrich-Alexander-Universität Erlangen-Nürnberg

Roderick PARKES, Stiftung Wissenschaft und Politik, Berlin

stellten die These ihrer gegenseitigen Wechselseitigkeit auf. Demnach sei es Anfang der 1990er Jahre in Form des Asylkompromisses auf der europäischen Ebene zur Europäisierung der deutschen Asylpolitik und damit zu einem ,top-down'-Prozess gekommen. Im Gegenzug hierzu sei aber auch im Sinne eines ,bottom-up'-Prozesses ein Politiktransfer von der nationalen, hier der bundesdeutschen, auf die europäische Ebene erfolgt, indem das deutsche Konzept der ,Sichere Drittstaaten“Regelung erfolgreich nach Europa exportiert wurde. Nach Lauter und Niemann können beide Transferprozesse (Europäisierungsprozesse) anhand von drei Analysefaktoren erklärt werden:

Den ersten Analysefaktor stelle die sicherheitsorientierte Agenda dar, denn die Analyse des Diskurses über die Asylpolitik habe gezeigt, dass diese Agenda den Diskurs sowohl in Deutschland schon in den 1990er Jahren als auch auf dem europäischen Level dominiert habe und somit der Durchsetzung des ,Sichere Drittstaaten'-Konzeptes zuträglich gewesen sei.

Als zweiten Analysefaktor, der dem Spiel mit den beiden politischen Handlungsebenen dienlich gewesen sei, nannten Lauter und Niemann das jeweilige institutionelle Gefüge: So habe das Einstimmigkeitserfordernis bei der Verabschiedung der Asylverfahrensrichtlinie ${ }^{3}$

3 Richtlinie 2005/85/EG des Rates vom 1. Dezember 2005 über die Mindestnormen für Verfahren in den Mitgliedstaaten zur Zuerkennung und Aberkennung der Flüchtlingseigenschaft, in: Amtsblatt der Europäischen Union, Nr. L 326 vom 13. Dezember 2005, S. 13-33. 
im Rat Deutschland in eine günstige Verhandlungssituation versetzt, in der seine Forderungen berücksichtigt werden mussten. Darüber hinaus konnte Deutschland auch erfolgreich auf nationalstaatliche Beschränkungen verweisen, da, so die damalige Argumentation der deutschen Verhandlungsführer, die Änderung des Grundgesetzes in Artikel 16 Absatz 2 erforderlich gewesen wäre, um der grundsätzlichen Vergemeinschaftung der Asylpolitik zustimmen zu können. Ergänzend sei von der rot-grünen Bundesregierung der Hinweis auf den schwarz-gelb dominierten Bundesrat hervorgebracht worden. Das parallel zur Verhandlung der Richtlinie in Deutschland behandelte Zuwanderungsgesetz habe der damaligen schwarz-gelben Mehrheit im Bundesrat als Argument dafür dienen können, die deutschen Forderungen in dem Richtlinienentwurf zu berücksichtigen. Die Kompromissfähigkeit sei demnach begrenzt gewesen, da erfolgreich auf innerstaatliche Barrieren rekurriert werden konnte.

Den dritten Analysefaktor stellten nach Lauter und Niemann exogene und funktionelle Faktoren dar. Im Falle der europäischen Migrationspolitik seien solche Faktoren das Prinzip der Personenfreizügigkeit - welches eine kompensatorische Maßnahme verlangt habe und der exogene Druck in Form des hohen Aufkommens von Asylsuchenden zu Beginn der 1990er Jahre. Beide Faktoren hätten als Rechtfertigung für eine restriktive Asylpolitik fungiert.

Daniela Kietz erläuterte in ihrem Beitrag den Vertrag von Prüm und ging dabei der Frage nach, ob es sich um ein Zweiebenenspiel oder eine Integrationsmethode nach dem Vorbild von Schengen handele. Die Entstehung und später erfolgte Umsetzung des Prümer Vertrags in europäisches Recht verdeutliche die Strategie einiger Mitgliedstaaten, die Europäisierung der Justiz- und Innenpolitik , durch die Hintertür " und anfänglich ohne Beteiligung der restlichen Mitgliedstaaten zu vollziehen. Ziel der Initiatoren des Prümer Vertrags sei es von Anfang an gewesen, den Vertrag in euro- päisches Recht zu überführen, so die These Kietz'. Die Methode der differenzierten Integration sei unter dem Vorwand gewählt worden, dass das Vorgehen innerhalb der dritten Säule ineffizient sei. Das Effizienzargument sei gegenüber dem Demokratieargument bevorzugt worden.

Aus demokratietheoretischer Sicht bestünden eine Reihe von Bedenken gegenüber diesem Vorgehen: Die parlamentarische Kontrollfunktion in Form des Mitentscheidungsverfahrens des Europäischen Parlamentes sei missachtet worden, ebenso habe eine Kontrolle der nationalen Parlamente nur durch die der ursprünglichen Prüm-Staaten stattgefunden. Des Weiteren seien Vertreter der Zivilgesellschaft nicht an der Ausarbeitung des Ratsbeschlusses beteiligt worden. Kietz stellte dem Effizienzargument gegenüber, dass durchaus andere Möglichkeiten der Integration innerhalb der dritten Säule wie die ,verstärkte Zusammenarbeit“ oder die Nutzung der Passerelle-Klausel zur Verfügung gestanden hätten. Das Effi-zienzargument habe lediglich als Vorwand für das gewählte Vorgehen gedient. Während es bei der Verhandlung der Schengen-Abkommen noch keine dritte Säule und keine entsprechenden Möglichkeiten des Voranschreitens unter Vermeidung des Einstimmigkeitserfordernisses gegeben habe, träfe dies nicht auf den Prümer Vertrag zu. Aus diesem Grund könne geschlussfolgert werden, dass die Akteure im Falle des Prümer Vertrags diese nachholende Integration intendiert hätten. Die Verhandlungsmethode entspreche mit Blick auf die institutionelle und rechtliche Ausgangssituation nur bedingt dem Schengener Integrationsprozess, wenngleich das Ergebnis beider Integrationsprozesse vergleichbar sei.

Liberalismus vs. Kontrolle: zur inhaltlichen Ausrichtung der europäischen Justiz- und Innenpolitik

Dass die Initiativen zur temporären Migration der Europäischen Kommission durch einen menschenrechtsbasierten sowie entwicklungs- 
politischen Ansatz ergänzt werden müssen, forderte Hendrik Cremer im zweiten Panel. Eine europäische Politik der temporären Migration solle mit Grundsätzen, wie sie unter anderem im sogenannten Palermo-Protokoll sowie im Europaratsprotokoll fixiert worden waren, konform sein. Der alleinige Fokus auf die Bedürfnisse der europäischen Arbeitsmärkte ebenso wie ein primär restriktiver Ansatz zur Verhinderung illegaler Migration, wie er prominent von Sarkozy und Schäuble in ihrem Strategiepapier 2006 vertreten worden war, würden den Herausforderungen und Chancen der temporären Migration nicht gerecht. Die schwierigen Verhandlungen mit Drittstaaten machten vielmehr deutlich, dass Möglichkeiten der legalen Migration und die Berücksichtigung der Vorteile für die Herkunftsstaaten Eingang in die Strategie finden müssten. Zugleich erkannte Cremer den Versuch der Europäischen Kommission an, die permanente und volkswirtschaftlich bedrohliche Abwanderung hoch- und mittelqualifizierter Fachkräfte und den damit einhergehenden ,brain drain“ aus gering entwickelten Herkunftsstaaten durch das Vorantreiben des Konzepts der zirkulären Migration zu verhindern. Doch liege es vielmehr an den Mitgliedstaaten, ein kohärentes Konzept der temporären Migration zu beschließen und zu verwirklichen. Die Steuerungsfähigkeit von Migration sei per se begrenzt und auch nur in bedingtem Maße durch Möglichkeiten der legalen Migration zu kontrollieren. Cremer warb deshalb dafür, Legislativmaßnahmen zur temporären Migration durch realistische Rückkehroptionen sowie durch die Sicherung von Rentenansprüchen für migrierte Drittstaatenangehörige zu ergänzen. Er schlussfolgerte, dass die Mitteilung ${ }^{4}$ zur ,zirkulären Migration“ einige Lösungsansätze zu Problemen wie Arbeitskräftemangel oder ,brain drain“ bereitstelle, dass jedoch die Förderung der zirkulären Migration weniger effektiv sein werde als erhofft. Es sei ratsam, migrations- und entwicklungspolitische Aspekte in anderen Politikbereichen zu stärken, um eine kohärente europäische Migrationspolitik zu erzielen.

Marie McGinley sprach sich in ihrem Beitrag gegen die Dichotomie von Liberalismus und Kontrolleffizienz innerhalb der dritten Säule aus und vertrat die These, dass die Integration eines rechtebasierten Ansatzes die Effektivität im Bereich des Datenschutzes erhöhe. Datenschutzrechte könnten einen Beitrag zu mehr Transparenz leisten und somit das Vertrauen zwischen den datenaustauschenden Parteien vergrößern. Bisher bestehe auf europäischer Ebene kein umfassender Datenschutz, der auch die polizeilich-justizielle Zusammenarbeit abdecken würde. McGinley wies jedoch auch darauf hin, dass das Haager Programm ${ }^{5}$ Datenschutzregelungen in der dritten Säule vorsehe. Momentan seien unterschiedliche Agenturen und Informationssysteme im Einsatz, ohne dass diese an die gleichen Datenschutzrechte gebunden seien. Der Rat habe im Falle des Rahmenbeschlussentwurfs ${ }^{6}$ zum Datenschutz von personenbezogenen Daten, die im Rahmen der polizeilichen und justiziellen Zusammenarbeit verarbeitet werden, Änderungsvorschläge des Europäischen Parlaments sowie von Fürsprechern des Datenschutzes wie den Europäischen Datenschutzbeauftragten nicht berücksichtigt. Der jüngste Entwurf zum Rahmenbeschluss könne jedoch kaum dazu beitragen, die Effektivität des Datenschutzes zu erhöhen, da der Umfang des zu regelnden Datenschutzes unklar bleibe. Die lange Verhandlungsdauer resultiere aus einer Reihe strittiger Fragen: Nicht geklärt sei, ob der innereuropäische Austausch von Daten oder lediglich der Austausch auf natio-

4 Europäische Kommission: Zirkuläre Migration und Mobilitätspartnerschaften zwischen der Europäischen Union und Drittstaaten, KOM(2007) 248 endgültig.

5 Rat der Europäischen Union: Tagung des Europäischen Rates (Brüssel, 4./5. November 2004), Schlussfolgerungen des Vorsitzes, Dok. 14292/1/04 REV 1 vom 8. Dezember 2004.

6 Rat der Europäischen Union: Proposal for a Council Framework Decision on the protection of personal data processed in the framework of police and judicial cooperation in criminal matters, Dok. 11365/3/07 vom 12. Oktober 2007. 
naler Ebene zu regeln sei. Uneinigkeit herrsche zudem über die Frage, ob auch nationale Daten dem Rahmenbeschluss unterlägen. Auch der Datenaustausch mit Drittstaaten stelle einen Konfliktpunkt dar. Nach Meinung von McGinley sei es zudem kritisch, wenn nachrichtendienstliche Tätigkeiten zum Zwecke der nationalen Sicherheit aus den Regelungen ausgenommen würden. Fraglich sei außerdem, wer die Angemessenheit von Datenschutzmaßnahmen, wie es in dem Entwurf angemahnt wird, beurteilen werde und wem die Kontrolle der Einhaltung obliege.

In ihrem Vortrag trat Ruth Weinzierl für eine menschenrechtskonforme Migrationspolitik der Europäischen Union ein. Sie bemängelte die menschenrechtliche Situation, der Immigranten insbesondere an den europäischen $\mathrm{Au}$ Bengrenzen ausgesetzt seien. Das Prinzip des ,non-refoulement" werde häufig nicht eingehalten. So seien Misshandlungen von Immigranten berichtet worden, ebenso wie die extraterritoriale Geltung von Menschenrechten infrage gestellt worden sei. Der Fokus der Europäischen Union richte sich wesentlich auf das Abfangen von Migranten vor Betreten der Territorien der Mitgliedstaaten. Somit drohe das in internationalen Verträgen verbriefte Verbot des ,refoulement" außer Kraft gesetzt $\mathrm{zu}$ werden, wenn Asylsuchende keinen $\mathrm{Zu}$ gang zu einem Asylverfahren bekämen. Neben solchen kritischen Aspekten seien jedoch auch positive Signale hinsichtlich des Menschenrechtsschutzes erkennbar. So könne die Europäische Union inzwischen auf ein harmonisiertes Flüchtlingsrecht verweisen. Von einem Triumph des kontrollorientierten Ansatzes könne daher nicht die Rede sein. Die (noch ausstehende) Bindung der Europäischen Union an die Grundrechtecharta sowie an die Europäische Menschenrechtskonvention führe zu Einschränkungen der staatlichen Souveränität. Dies seien wesentliche Voraussetzungen für die Wahrung menschenrechtlicher Ansprüche. Es müsse jedoch weiterhin systematisch die (Teil-)Harmonisierung der Asylpolitik durch menschenrechtliche Standards vervollständigt werden, so Weinzierl.
Deutschland als Akteur der europäischen Justiz- und Innenpolitik

Deutschland habe eine herausragende Rolle bei der Entwicklung der europäischen Grenzschutzagentur FRONTEX gespielt, stellte Mechthild Baumann in ihrem Vortrag fest. Der von Deutschland initiierte Aufbau von FRONTEX sei als eine späte Ausgleichsmaßnahme für den primär von Deutschland empfundenen Souveränitätsverlust durch den Schengen-Raum zu werten. Ziel sei einerseits gewesen, die verlorengegangene Kontrollfähigkeit wieder zu erlangen. Andererseits sei aber auch Personalpolitik betrieben worden, denn der Abbau innereuropäischer Grenzkontrollen habe zu der Notwendigkeit geführt, das hierdurch frei gewordene Personal anderweitig einzusetzen. Nicht zuletzt habe der Aufbau eines europäischen Grenzschutzregimes auch Züge symbolischer Sicherheitspolitik gegenüber der von der Öffnung der Grenzen beunruhigten deutschen Bevölkerung getragen. FRONTEX könne als Beispiel der kontrollorientierten Politik gelten, sei jedoch auch im historischen Kontext zu sehen, da Deutschland Anfang der 1990er Jahre von einem anschwellenden Flüchtlingsstrom betroffen gewesen sei. FRONTEX sei insofern als Ergebnis von, venue-shopping ' zu werten, als ein Ausweichen auf die europäische Ebene von deutscher Seite vorangetrieben worden sei, ohne Kompetenzen zu Migrationsfragen zum Beispiel beim Bundesinnenministerium einzuschränken. Baumann verneinte jedoch die Annahme, dass es sich bei der Entwicklung von FRONTEX um eine Exit-Strategie handele. Im Gegensatz hierzu sei jedoch die Initiative der „High Level Group on the Future of European Home Affairs" während der deutschen Ratspräsidentschaft der Europäischen Union im ersten Halbjahr 2007 als klassische Exit-Strategie zu werten: Geschützt vor der Öffentlichkeit und parlamentarischer Kontrolle werde dort die Zukunft der Grenzpolitik für die Zeit nach Ablauf des Haager Programms skizziert. 
Ob und wie die deutsche Exekutive als, venue-shopper' im Bereich der europäischen Justiz- und Innenpolitik agiere, analysierte $\mathrm{Si}$ mon Bulmer. Deutschland sei als starker Förderer der Vergemeinschaftung der Justiz- und Innenpolitik aufgetreten, wobei es transgouvernementale Kooperationsformen bevorzuge, um eigenen Präferenzen erfolgreich einbringen zu können. Dabei läge in Deutschland eine ,schizophrene" Ausgangslage vor: Auf der einen Seite bestünden starke institutionelle Barrieren wie der deutsche Föderalismus und die Beteiligung einer relativ hohen Anzahl unterschiedlicher Bundesministerien an der Gestaltung der Justiz- und Innenpolitik. Andererseits verfüge insbesondere das Bundeskanzleramt über einen großen Handlungsspielraum bei der Durchsetzung seiner Interessen. Das Konzept des ,venue-shopping' sei für Deutschland nur bedingt anwendbar, da Deutschland zugleich auch als Befürworter der differenzierten Integration wie beim Vertrag von Prüm, der Kooperation mit den „G6“ oder der „Future Group“ auftrete. Gegen die ,venue-shopping '-These spräche zudem, dass sich Deutschland gegen die Ausweitung der qualifizierten Mehrheitsentscheidungen stelle. Dies weise daraufhin, dass Deutschland kein vorbehaltsloser Befürworter der Vergemeinschaftung der Migrationspolitik sei. Bulmer betrachtete das, venueshopping ' weniger als vollständig rational gesteuerte Strategie, denn als ein (zufälliges) Handeln ohne strategisches Abschätzen der Konsequenzen des, venue-shopping'. Dies erkläre die widersprüchliche Position Deutschlands bei der Harmonisierung. Offen bleibe, so Bulmer, wie die ,venue-shopping'-These, wenn sie als Alternative zu Integrationstheorien wie dem liberalen Intergouvernementalismus und dem (Neo-)Funktionalismus betrachtet würde, die Vergemeinschaftung nicht nur von , low politics' sondern auch von, high politics' - wie es die Justiz- und Innenpolitik in weiten Teilen darstelle - erklären könne.

Deutschlands Antiterrorpolitik war Gegenstand von Wilhelm Knelangens Vortrag. Deutschland ebenso wie andere europäische
Mitgliedstaaten verfolgten innerhalb dieses Politikfeldes in erster Linie einen kontrollorientierten Ansatz. Dieser sei insbesondere durch die terroristischen Anschläge des 11. September 2001 gerechtfertigt worden. Innerhalb Deutschlands sei zu beobachten, dass polizeiliche und nachrichtendienstliche Kompetenzen erweitert worden seien. So seien bürgerrechtliche und menschenrechtliche Positionen durch eine sicherheitsorientierte Agenda in den Hintergrund gedrängt worden. Für diese Entwicklung machte Knelangen die Dynamik des deutschen Parteienwettbewerbs verantwortlich. De facto bestehe in Deutschland seit den 1970er Jahren - bedingt durch das deutsche Modell des kooperativen Föderalismus - im Bereich der Sicherheitspolitik eine Große Koalition. Verfassungsänderungen seien durch das Erfordernis einer Zweidrittelmehrheit nur innerhalb einer faktischen GroBen Koalition zu erzielen. CDU wie SPD besetzten Themen der Sicherheitspolitik jeweils für sich. Liberale Ansätze könnten entweder von der Opposition nicht effektiv eingebracht werden oder würden von dem jeweils anderen Koalitionspartner nicht unterstützt. Bürgerund menschenrechtliche Gruppierungen hätten zudem keine Einflussmöglichkeiten im Entscheidungsprozess. Eine menschenrechtliche Dimension in der deutschen Sicherheitspolitik gestalte sich vielmehr als Debatte über das Verfassungsrecht: Die deutsche Verfassungsgerichtsbarkeit trete damit als Gegenspieler der zunehmenden, Versicherheitlichung' auf. Zentrale Bereiche der Antiterrorgesetzgebung seien von ihr eingeschränkt worden. Auch trete das Bundesverfassungsgericht als Akteur auf, welcher die Menschenwürde gegenüber Sicherheitsinteressen betone. Die These, dass es sich beim deutschen politischen Handeln um ,venue-shopping" handele, relativierte Knelangen. Primär ginge es in der Antiterrorpolitik um Handeln auf nationaler Ebene; die dominante Position der Bundesregierung beim Agenda-Setting und der Repräsentation Deutschlands auf der europäischen Ebene werde durch den eher passiven Bundestag kaum beeinträchtigt. Da es dem EU-Recht eigen sei, die Bedingungen der Kooperation und 
Koordination vorauszusetzen, sei ,venueshopping' eher unwahrscheinlich. Vielmehr resultiere die faktische Autonomie der Bundesregierung in diesem Bereich aus den Regeln des Parteienwettbewerbs.

Europäisierungsmodelle in der Justiz- und Innenpolitik - Reflexionen über Theorie- und Analysekonzepte

Ausgehend vom ,misfit"-/,top-down'-Modell ging Timm Beichelt der Frage nach, ob dieser Strang der Europäisierungsforschung auch für den Bereich Justiz und Inneres fruchtbar sei. Beichelt vertrat die These, dass im Bereich der europäischen Justiz- und Innenpolitik politischer Wandel eher in geringem Maße EUinduziert sei, da hier vornehmlich ein konsensualer Entscheidungsmodus festgeschrieben sei. Es bestünden innerstaatlich eine Vielzahl von Vetopositionen - im deutschen Falle sei dies beispielsweise der Bundesrat -, sodass das nationale Level als intervenierende Ebene charakterisiert werden könne. Beichelts Alternativvorschlag für ein Europäisierungsmodell stellt ein Transformationsmodell dar, welches eine Vielzahl von einwirkenden Vektoren einbeziehen kann. Wandel im Bereich Justiz und Inneres sei tendenziell binnenstaatlich motiviert und der europäische Einfluss eher nachgeschaltet. Die europäische Ebene löse damit Wandel nicht aus, sondern verändere vielmehr den binnenstaatlichen Prozess.

Anhand einer (analytischen) Analogie der Entwicklung des europäischen Binnenmarktes mit derjenigen der europäischen Justizund Innenpolitik beschrieb Wolfgang Wagner die Integrationsprozesse des Politikfelds der Inneren Sicherheit. Entsprechend der Analogie unterschied er dabei zwischen positiver und negativer Integration. Vergemeinschaftungsmaßnahmen, die der positiven Integration zuzurechnen seien, seien durch langwierige Entscheidungsverfahren und Ergebnisse, die dem kleinsten gemeinsamen Nenner entsprechen, gekennzeichnet. Solche Re-Regulierungen durch Mindeststandards ließen sich bei Grundrechten oder beim Datenschutz fin- den. In der überwiegenden Mehrheit seien im Bereich der Inneren Sicherheit Maßnahmen zu beobachten, die Hindernisse in der Vergemeinschaftung abschafften und dem Ziel eines gemeinsamen Marktes beziehungsweise eines europäisch harmonisierten gemeinsamen strafjustiziellen Raumes dienten. Auch negative Integrationsmaßnahmen würden zunächst versuchen, die Rechtsvorschriften anzugleichen, erreichten jedoch meist nach langen Entscheidungsverfahren nur Ergebnisse, die einem Minimalkonsens entsprächen. Daraufhin folgten, so Wagner, zumeist Prinzipien der gegenseitigen Anerkennung als Integrationsinstrument. Die gegenseitige Anerkennung führe zu beschleunigten Entscheidungsverfahren sowie zu einer weitreichenden gemeinsamen Steuerung innerhalb eines Politikfeldes. Wagner illustrierte das Konzept der positiven und negativen Integration am Beispiel des europäischen Haftbefehls und Europol. Die Unterscheidung zwischen negativer und positiver Integration helfe, Durchsetzungschancen sicherheitsbezogener und freiheitssichernder Initiativen zu antizipieren und stehe in Ergänzung zur , venue-shopping 'These sowie diskurstheoretischer Ansätze.

\section{Fazit}

Mittels verschiedener, durchaus komplementärer Erklärungsansätze aus der Integrationstheorie sowie akteurs- und spieltheoretischen Herangehensweisen gelang es auf der Tagung, das breite Spektrum an sub-policies im „Raum der Freiheit, der Sicherheit und des Rechts“ zu analysieren und die in diesem Raum existenten Widersprüche zwischen Souveränitätsstreben und Vergemeinschaftung, zwischen Liberalismus und Kontrolle zu erhellen. Ergänzend dazu wurden auch policy-analytische Ansätze als nutzbringend für die Analyse qualifiziert, da diese auch nichtintendierte Variablen in den Blick nehmen.

Die Veranstalter dieser Tagung beabsichtigen, diese Ergebnisse in Form eines Bandes herauszugeben. 